\title{
La salud de los trabajadores hospitalarios desde el punto de vista psicosocial
}

\section{The health of hospital workers from the psychosocial point of view}

\author{
Mg. María Cristina Abril Freire ${ }^{1}$ \\ mariacabril@uta.edu.ec \\ Mg. Iveth Katherine Sánchez Carvajal ${ }^{2}$ \\ ikscarvajal@msn.com
}

Recibido: 1/09/2017, Aceptado: 1/11/2017

\begin{abstract}
RESUMEN
La relación entre trabajo y salud puede abordarse desde distintos ámbitos, en el presente estudio se aborda desde la perspectiva psicosocial, este proceso se debe analizar en todas las actividades humanas de la cual depende la construcción de la sociedad y tiene una vital relación intencional dentro del quehacer organizacional hospitalario, de aquí parte la importancia de investigar la salud de los trabajadores del área de Consulta Externa (CE) del Hospital General Docente Ambato, así, determinar las debilidades que permitan evaluar el riesgo. Se utilizó un enfoque holístico de corte transversal, en este caso, se coartó un alcance descriptivo, identificando si hay intencionalidad de respuesta, un error de comprensión o una interpretación subjetiva de la realidad, todo esto en un método de detalle y descripción con la información de las cuatro variables que estudia el Cuestionario de Evaluación de Factores Psicosociales que identifican situaciones de riesgo, relacionadas en gran manera con el entorno laboral y elementos que afectan a la salud del trabajador. Finalmente, los resultados mostraron un estado adecuado en las cuatro dimensiones de estudio, pero con un bajo nivel en la dimensión de comunicación y gestión de tiempo, variables que se aconseja estudiar a profundidad para mejorar las relaciones intra e interpersonales de los trabajadores de CE.
\end{abstract}

Palabras clave: Identificación de riesgo, hospital, cuestionario factores psicosociales, salud laboral

\section{ABSTRACT}

The relationship between work and health can be studied from different angles, in this study it is taken from the psychosocial perspective, this process must be present in all human activities on which the construction of society depends and has a vital intentional relationship within the hospital organizational tasks, hence the importance of investigating the internal communication and its link with the work efficiency of the staff of the External Consultation of the Ambato General Teaching Hospital to

\footnotetext{
${ }^{1}$ Docente de la Universidad Técnica de Ambato. Ecuador

${ }^{2}$ Hospital General Docente Ambato. Ecuador
} 
बत

No. 17, 31 de enero de 2018

ISSN impreso: 1390 - 6321

determine the weaknesses and strengths that make it possible to generate strategies to foster communicative actions that generate a productive, harmonious and participative environment that allows to improve the work efficiency. The relationship between the internal communication and the work efficiency of the External Consultation staff of General Ambato Hospital is determined by analyzing the factors that affect the internal communication in the Hospital, identifying the level of Labor efficiency of staff and the correlation between internal communication and the statistical correlation between both study factors.

Keywords: Risk identification, hospital, psychosocial factors survey, work health

\section{Introducción}

Los hospitales son sistemas complejos que, como ninguna otra organización, deben mantener un nivel muy alto de comunicación en todos sus niveles, áreas, secciones y departamentos para mantener fluidez en un trabajo exigido como un derecho de acceso público oportuno sin exclusión. Formanchuk (2010) cita a Peter Drucker, el teórico del Management, señala: "el $60 \%$ de los problemas empresariales son consecuencia de una mala comunicación".

El Hospital General Docente Ambato está conformado por tres áreas: Emergencia, Hospitalización y Consulta Externa (CE); esta última atiende turnos para consultas médicas, psicológicas y odontológicas. Cuenta con alrededor de 54 profesionales médicos, que atienden de lunes a viernes en horarios de 7:00 a 19:00 y los días sábados de 7:00 a 15:30.

En el área de CE existen algunos problemas generados por la comunicación informal o rumores lo cual ocasiona distorsión del mensaje que se emite, esto se debe a que las actividades y funciones no están delegadas correctamente, cada funcionario procede a realizar sus funciones de acuerdo al mensaje o instrucción que recibe ese rato, generando cuellos de botella, y retrasos en las actividades que estaban planificadas, lo que conlleva a problemas entre los colaboradores, en su forma de trabajar, afectando la eficiencia laboral.

La comunicación en esta institución de salud, como en las demás instituciones del Ministerio de Salud del Ecuador, es manejada a través del correo electrónico institucional (ZIMBRA) y del sistema de gestión documental (QUIPUX) por medio de los cuales "se envía y se recibe oficios, memorandos, circulares y todo lo que implica comunicación formal dentro y fuera de la institución" (Secretaría Nacional de la Administración Pública, 2015).

Lamentablemente, la mayoría de colaboradores no tienen cuenta personal en el Quipux, siendo de uso únicamente para los directivos. El sistema Zimbra, por otro lado, no es utilizado diariamente por los colaboradores debido a la falta de capacitación para el uso de estas herramientas informáticas de comunicación directa; por esta razón, el personal de CE, desconoce las funciones encomendadas y no se entera de lo que sucede con la institución a nivel local y más aún a nivel nacional.

Además, el hecho de no poder agendar turnos por la falta de profesionales médicos, alarga los días de espera en busca de una cita médica de especialidad, generando un aumento de reclamos por la insatisfacción del paciente contaminando esa 
negatividad, ira, y maltrato en algunas veces, por supuesto, no queda otro efecto más claro en el área de CE un clima de tensión, estrés y de conflicto laboral que bloquea la comunicación interna y por lo tanto la eficiencia laboral del equipo de trabajo.

La relación entre comunicación interna y la eficiencia laboral en el Hospital General Docente Ambato se puede percibir muy distante, lo cual genera desconfianza en la prestación de sus servicios aun cuando se está repotenciando la infraestructura y equipamiento médico.

Por tanto, la comunicación interna y su relación con la eficiencia laboral en CE del Hospital General Docente Ambato se debe al deficiente uso de los medios de comunicación interna lo cual genera ineficiencia laboral, reflejada en la poca capacidad de disponer de los recursos necesarios para la toma oportuna de decisiones.

\section{Desarrollo}

La comunicación institucional externa e interna hospitalaria constituye una actividad de reciente creación impulsada permanentemente a través de varios factores, externamente Medina (2012) afirma que el desarrollo de las nuevas tecnologías de la comunicación, el surgimiento de los grupos hospitalarios privados o el interés creciente que la gente manifiesta hacia la salud, han obligado a los directivos a innovar en sistemas de comunicación como herramienta directiva con valor estratégico (p. 19), e internamente, es uno de los aspectos organizacionales que más importancia e impacto tiene, ya que las instituciones han comenzado a considerarla como una "herramienta estratégica capaz de influir de forma positiva en el buen funcionamiento de un hospital".

Un hospital sin una buena comunicación interna y externa simplemente está destinado al fracaso ya que no existe una adecuada articulación de todas sus áreas, tendientes a generar productos y servicios orientados a la promoción de la salud del ciudadano para satisfacer sus necesidades.

A criterio de Mira (2013), los hospitales son una fuente inagotable de información interna; por su parte, Quino (2014) reconoce que la comunicación interna en el Hospital Harry Williams de Cochabamba, Bolivia, es vital en la intención de ofrecer un servicio de calidad y de crecimiento organizacional basados en un marco de excelencia que orienta el cumplimiento de los objetivos institucionales.

Herrero (2015) realizó un estudio comparativo de eficiencia técnica de los hospitales públicos y de las empresas públicas hospitalarias de Andalucía, España; señalando que las empresas públicas poseen mayor eficiencia atribuida al buen manejo de la comunicativo horizontal y vertical que forman un todo indisoluble por las interrelaciones que producen.

Consecuentemente, la comunicación se torna en un elemento integral dentro de los factores que forman para de la eficiencia laboral dentro de un servicio de salud ya que favorece la cohesión y actitudes de coherencia en las tareas que implican los componentes organizacionales y que generan nuevos comportamientos mentales y de lenguaje común para el trabajo en equipo. 
Suing (2012) afirma que: "En el Ecuador se vive un proceso de cambios emergentes a consecuencia de las nuevas leyes; las instituciones ecuatorianas revisan sus prácticas, modifican órganos y evalúan su pertinencia social. Las transiciones demandan fortalecer sus capacidades y para ello proyectar la comunicación interna es urgente".

Por ello, las instituciones hospitalarias como entes públicos, se encuentran en un proceso de acreditación internacional que avale la calidad del servicio a través de la eficiencia laboral de sus colaboradores.

Según el Banco Central del Ecuador (2010):

"El país se ubicó en el puesto 101 del Índice de Competitividad Global (ICG) de entre 139 países. Los resultados de esta medición fueron difundidos por el Foro Económico Mundial, que señala que el país permanece rezagado en el ámbito de la competitividad, con una leve tendencia al alza. Otros datos preocupantes en la medición se encuentran en la categoría de eficiencia laboral, donde el Ecuador se situó en el puesto 137 de los 139 países analizados."

La comunicación interna ha sido considerada hasta ahora un paradigma que no requiere de gestión y de procesos para que genere un entorno productivo, armonioso y participativo.

En Ecuador, utilizar los servicios de hospitales públicos constituye un verdadero reto para el usuario, las quejas y reclamos constantes por la falta de información oportuna las consultas médicas generan insatisfacción en el usuario lo cual influye negativamente en la eficiencia laboral y consecuentemente en la imagen institucional.

Se evidencian fuertes flujos de comunicación informal que generan desorganización en el trabajo, ya que un mismo colaborador recibe diferentes órdenes de manera informal al mismo tiempo, creando confusión, equivocación, desorden en la ejecución de su trabajo que lamentablemente afecta su eficiencia laboral. Si los flujos de comunicación provenientes de cualquier nivel no circulan por canales formales con transparencia y velocidad pertinente, se produce vacíos de información que son llenados por rumores negativos que desvían la atención de la gestión organizacional.

La comunicación es un proceso presente en todas las actividades humanas de la cual depende la construcción de la sociedad y tiene una vital relación intencional dentro del quehacer organizacional hospitalario de ahí la importancia de investigar la comunicación interna y su vinculación con la eficiencia laboral del personal de CE para determinar las debilidades y fortalezas que posibiliten generar estrategias para potenciar acciones comunicativas que generen un entorno productivo, armonioso, participativo que permita mejorar la eficiencia laboral.

\section{Metodología}

Dentro del abordaje de la investigación se determina la relación entre la comunicación interna con la eficiencia laboral del personal de CE, a través del análisis de los factores que afectan la comunicación interna en el Hospital, la identificación del nivel de eficiencia laboral del personal y la correlación que existe entre la comunicación interna y la correlación estadística entre ambos factores de estudio. Por tanto, el enfoque fue 


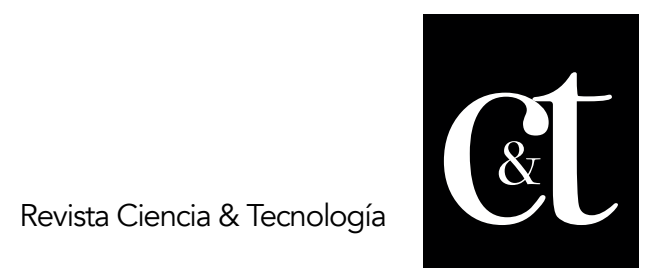

No. 17, 31 de enero de 2018

ISSN impreso: 1390 - 6321

mixto (cuali-cuantitativo) porque se basa "en métodos de recolección de datos y confía en la medición numérica, el conteo y el uso de la estadística descriptiva para el análisis e interpretación de resultados" (Gómez, 2006).

Se emplearon dos modalidades de estudio: documental y de campo, medios de información a través de los que se analizó la información descrita y posteriormente de forma sistemática de los hechos en el lugar donde se producen, el alcance fue descriptivo - correlacional que tuvo como propósito examinar la relación entre las variables y a su vez medir el grado de relación que existe entre ellas; es decir, en qué medida influye la comunicación interna en la eficiencia laboral en el área de CE del Hospital General Docente Ambato.

\section{Población}

La población de estudio estará conformada por el personal que labora en $\mathrm{CE}$, cuya distribución se describe en la tabla a continuación.

\section{Tabla 1. Población}

\begin{tabular}{|l|l|}
\hline DESCRIPCIÓN & CANTIDAD \\
\hline Gerente & 1 \\
Administrativos & 23 \\
Médicos tratantes & 54 \\
Psicólogos & 2 \\
Enfermeras & 6 \\
Auxiliares de Enfermería & 5 \\
Tecnólogos Médicos & 5 \\
\hline TOTAL: & 96 \\
\hline
\end{tabular}

Fuente: Elaboración propia

Por tratarse de un número finito y menor a 100, se trabajó con el total de la población y no se calculó el tamaño de la muestra.

Como instrumentos de Recolección de la Información se utilizó el Test de Factores Psicosociales (Identificación de situaciones de riesgo), con una validez de contenido y de constructo muy amplia, que fue sometido a revisión aplicando varios métodos de evaluación de riesgos psicosociales como lo demuestra el trabajo de Nogareda (2009) señalando que sus características psicométricas y valores de referencia son positivos en cuanto a validez y fiabilidad con respecto a otros tests utilizados como: 
- El WOCCQ (Cuestionario de condiciones de trabajo y control), método belga de diagnóstico de riesgos psicosociales desarrollado por el Servicio de Psicología del Trabajo y de las Empresas de la Universidad de Lieja.

- Las listas de Control de la Fundación para la Mejora de las Condiciones de Vida y de Trabajo (Kompier y Levi, 1994).

- El Método de evaluación de factores psicosociales del Instituto de Seguridad e Higiene en el Trabajo de España - INSHT respecto a la validez de constructo y a la fiabilidad.

Para llegar al actual cuestionario se han llevado a cabo distintas fases de análisis.

Cada uno de estos instrumentos citados anteriormente presentan un conjunto de factores humanos del medio ambiente laboral que están en continua interacción con la dinámica laboral y recoge las reacciones y consecuencias de los colaboradores con respecto a sus necesidades, cultura, habilidades, vulnerabilidad, capacidad de adaptación y vida privada que afectan a la motivación, la insatisfacción en el trabajo que genera estrés; estos aspectos dependen de la percepción que el personal tenga y de la capacidad para hacer frente a los mismos.

El Test de Factores Psicosociales, Identificación de Situaciones de Riesgo, a criterio de Bastida (2013) posee las siguientes características en cuanto a su validez, confiabilidad y fiabilidad. 


\section{Tabla 2. Características particulares del Test de Factores Psicosociales, Identificación de Situaciones de Riesgo}

\begin{tabular}{|c|c|}
\hline $\begin{array}{l}\text { Población a la que se } \\
\text { dirige }\end{array}$ & $\begin{array}{l}\text { Total de colaboradores de la entidad especialmente en el } \\
\text { caso de empresas pequeñas. Se recomienda en el manual } \\
\text { de administración el muestro en las grandes entidades. }\end{array}$ \\
\hline $\begin{array}{l}\text { Estructura del } \\
\text { Instrumento de Medida }\end{array}$ & $\begin{array}{l}\text { Se trata de un cuestionario convencional, estructurado de } \\
\text { forma multifactorial en los siguientes factores de medición: } \\
\text { Participación, implicación y } \\
\text { Responsabilidad } \\
\text { Formación, información y comunicación. } \\
\text { Gestión del tiempo. } \\
\text { Cohesión de grupo. }\end{array}$ \\
\hline Medidas que aportan & $\begin{array}{l}\text { Se obtiene una puntuación, en cuatro niveles de riesgo, para } \\
\text { cada uno de los factores descritos que identifican la situación } \\
\text { de grupos de colaboradores en riesgo. }\end{array}$ \\
\hline $\begin{array}{l}\text { Modelo Psicométrico } \\
\text { de Construcción del } \\
\text { Instrumento de } \\
\text { Evaluación }\end{array}$ & $\begin{array}{l}\text { El cuestionario aquí valorado se ha generado a partir de la } \\
\text { Teoria Clásica de los Test con norma de baremación de } \\
\text { grupo. }\end{array}$ \\
\hline $\begin{array}{l}\text { Datos de datos de } \\
\text { validez y fiabilidad } \\
\text { psicométricas }\end{array}$ & $\begin{array}{l}\text { Existen diversos estudios a propósito de la validez y fiabilidad } \\
\text { de este cuestionario, todos ellos confirman la estructura } \\
\text { multifactorial del cuestionario y ofrecer valores de Alfa de } \\
\text { Cronbach (fiabilidad) elevados. Su uso está garantizado } \\
\text { desde el punto de vista psicométrico. }\end{array}$ \\
\hline Ventajas & $\begin{array}{l}\text { Se trata de un instrumento muy sencillo, fácil de administrar } \\
\text { y sin especiales peculiaridades. Requiere poco tiempo, pocas } \\
\text { instrucciones y puede ser de administración colectiva. }\end{array}$ \\
\hline
\end{tabular}

\section{Resultados y discusión}

La primera dimensión que brinda el test anteriormente descrito denominado, Participación, Implicación, Responsabilidad que se refiere al grado de libertad e independencia para controlar y organizar su propio trabajo, así como para determinar los métodos a utilizar (Lahera y Góngora, 2002). 
Tabla 3. Dimensión 1

\begin{tabular}{|l|l|l|}
\hline ESTADO & RANGO & FRECUENCIA \\
\hline Muy Inadecuado & $27-44$ puntos & 0 \\
\hline Inadecuado & $18-26$ puntos & 46 \\
\hline Adecuado & $9-17$ puntos & 39 \\
\hline Muy Adecuado & $0-8$ puntos & 11 \\
\hline TOTAL=> & & 96 \\
\hline
\end{tabular}

Fuente: Resultados Test Factores Psicosociales

De los 96 colaboradores, 46 corresponden al $48 \%$ y se encuentran en estado inadecuado; 39 colaboradores, correspondiente al $41 \%$ se encuentran en estado adecuado, mientras que apenas 11 colaboradores equivalente al $11 \%$ están en el estado muy adecuado. Consecuentemente, los encuestados evidencian un estado inadecuado en la dimensión autonomía, trabajo en equipo, iniciativa, control sobre la tarea, rotación, supervisión y enriquecimiento de tareas.

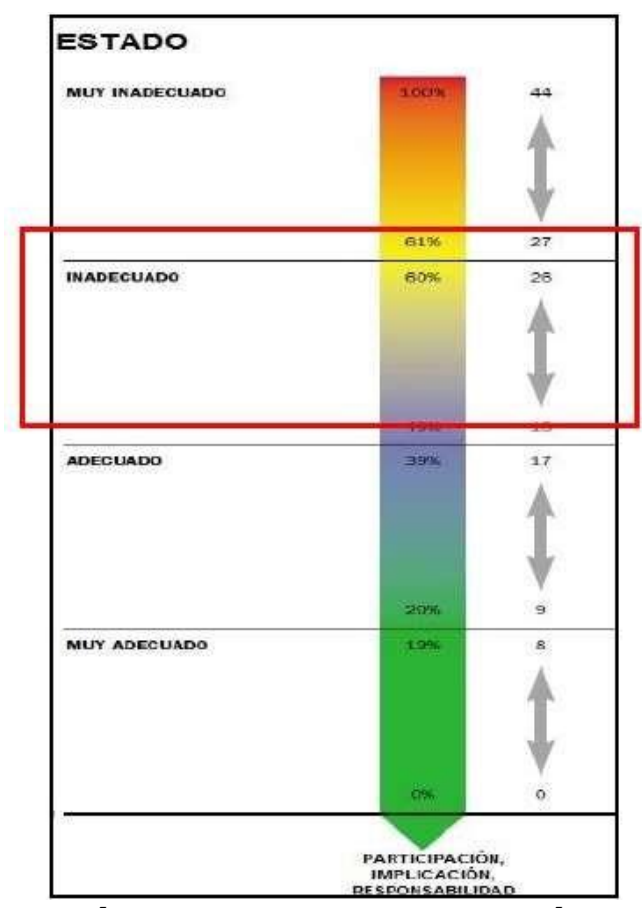

Gráfico 1. Estado Dimensión 1

Fuente: Tabla 3 
La dimensión participación, implicación, responsabilidad detalla el grado de libertad e independencia que tiene el colaborador para controlar, organizar su trabajo y determinar los métodos a utilizar.

La segunda dimensión del test denominada, Formación, Información, comunicación, se refiere al grado de interés personal que la organización demuestra a los funcionarios.

Tabla 4. Dimensión 2

\begin{tabular}{|l|l|l|}
\hline ESTADO & RANGO & FRECUENCIA \\
\hline Muy Inadecuado & $22-35$ puntos & 19 \\
\hline Inadecuado & $14-21$ puntos & 48 \\
\hline Adecuado & $7-13$ puntos & 18 \\
\hline Muy Adecuado & $0-6$ puntos & 11 \\
\hline TOTAL=> & & 96 \\
\hline
\end{tabular}

Fuente: Resultados Test Factores Psicosociales

La dimensión formación, información y comunicación establece el interés que la institución demuestra por sus colaboradores, a través del flujo de información para delimitar bien sus funciones, el cumplimiento de su trabajo garantizando la óptima correlación entre el puesto de trabajo y las personas que lo ocupan. De los 96 encuestados, el $20 \%$ se encuentran en el estado muy inadecuado, el $50 \%$ se encuentra en el estado inadecuado, mientras tanto que el $19 \%$ están en el estado adecuado; y finalmente el $11 \%$ se encuentran en el estado muy adecuado.

De acuerdo a los resultados obtenidos, la mayoría de colaboradores evidencia una inadecuada formación, información y comunicación interna como consecuencia del deficiente uso de medios de comunicación interna, desconocimiento de perfiles de cargos según niveles jerárquicos, fuertes flujos de comunicación informal y escasa difusión de la planificación institucional. 


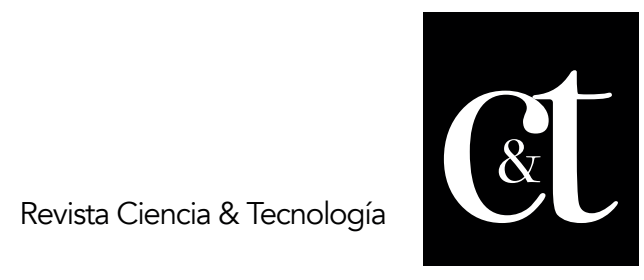

No. 17, 31 de enero de 2018

ISSN impreso: 1390 - 6321

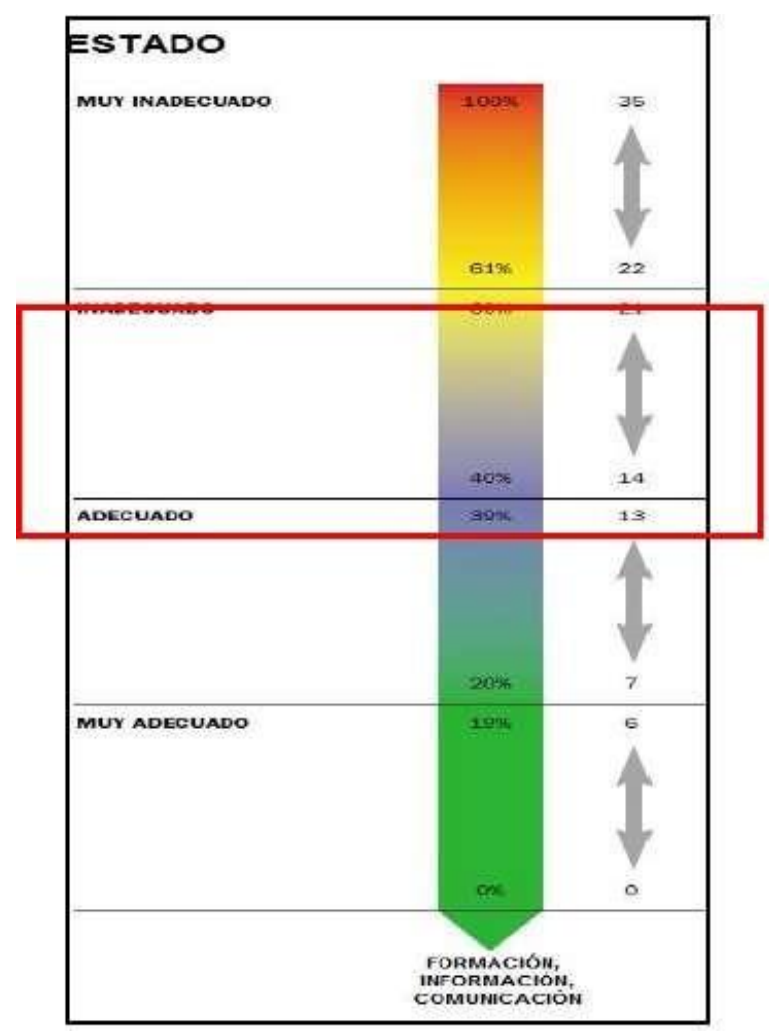

Gráfico 2. Estado Dimensión 2

Fuente: Tabla 4

La tercera dimensión es Gestión del Tiempo que corresponde al Nivel de autonomía concedida al funcionario para determinar su ritmo de trabajo, la distribución de las pausas y las vacaciones de acuerdo a sus necesidades personales.

Tabla 5. Dimensión 3

\begin{tabular}{|l|l|l|}
\hline ESTADO & RANGO & FRECUENCIA \\
\hline Muy Inadecuado & $15-24$ puntos & 22 \\
\hline Inadecuado & $10-14$ puntos & 39 \\
\hline Adecuado & $5-9$ puntos & 26 \\
\hline Muy Adecuado & $0-4$ puntos & 9 \\
\hline TOTAL=> & & 96 \\
\hline
\end{tabular}

Fuente: Resultados Test Factores Psicosociales 
La dimensión gestión del tiempo establece el nivel de autonomía que la institución otorga a cada uno de sus colaboradores para determinar el ritmo, carga y fatiga en el trabajo. De los 96 colaboradores encuestados, el $23 \%$ se encuentran en el nivel muy inadecuado, el $41 \%$ están en el estado inadecuado, el $27 \%$ están en el estado adecuado mientras que solamente 9 colaboradores equivalente al $9 \%$ están en el estado muy adecuado de la dimensión 3 gestiones del tiempo.

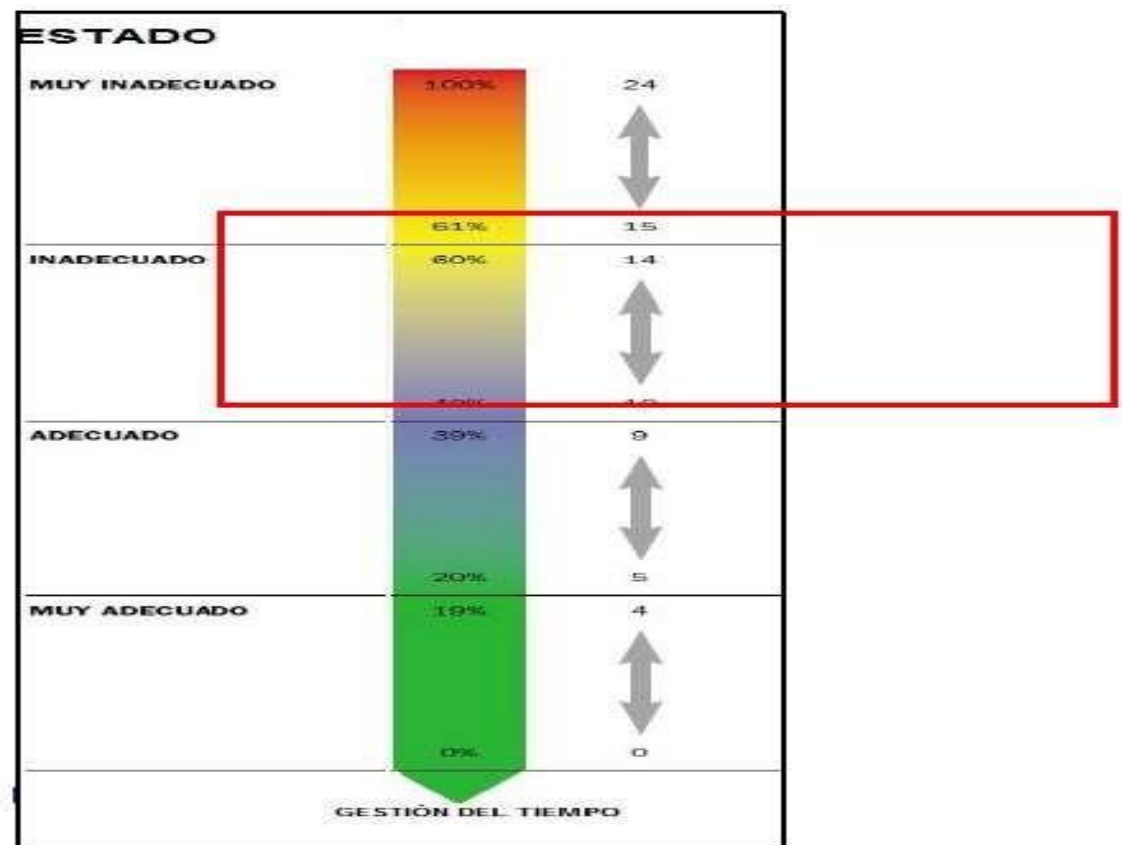

Gráfico 3. Estado Dimensión 3

Fuente: Tabla 5

Los resultados obtenidos evidencian la existencia de colaboradores que no tienen autonomía en su trabajo debido a la división de trabajo para elevar su eficiencia optimizando los recursos disponibles. Los colaboradores que se encuentran en el estado muy adecuado son los líderes departamentales.

Cuarta dimensión denominada cohesión de grupo que define la cohesión como patrón de estructura del grupo de trabajo. 


Tabla 6. Dimensión 4
\begin{tabular}{|l|l|l|}
\hline ESTADO & RANGO & FRECUENCIA \\
\hline Muy Inadecuado & $18-29$ puntos & 0 \\
\hline Inadecuado & $11-17$ puntos & 74 \\
\hline Adecuado & $6-10$ puntos & 19 \\
\hline Muy Adecuado & $0-5$ puntos & 3 \\
\hline TOTAL=> & & 96 \\
\hline
\end{tabular}

Fuente: Resultados Test Factores Psicosociales

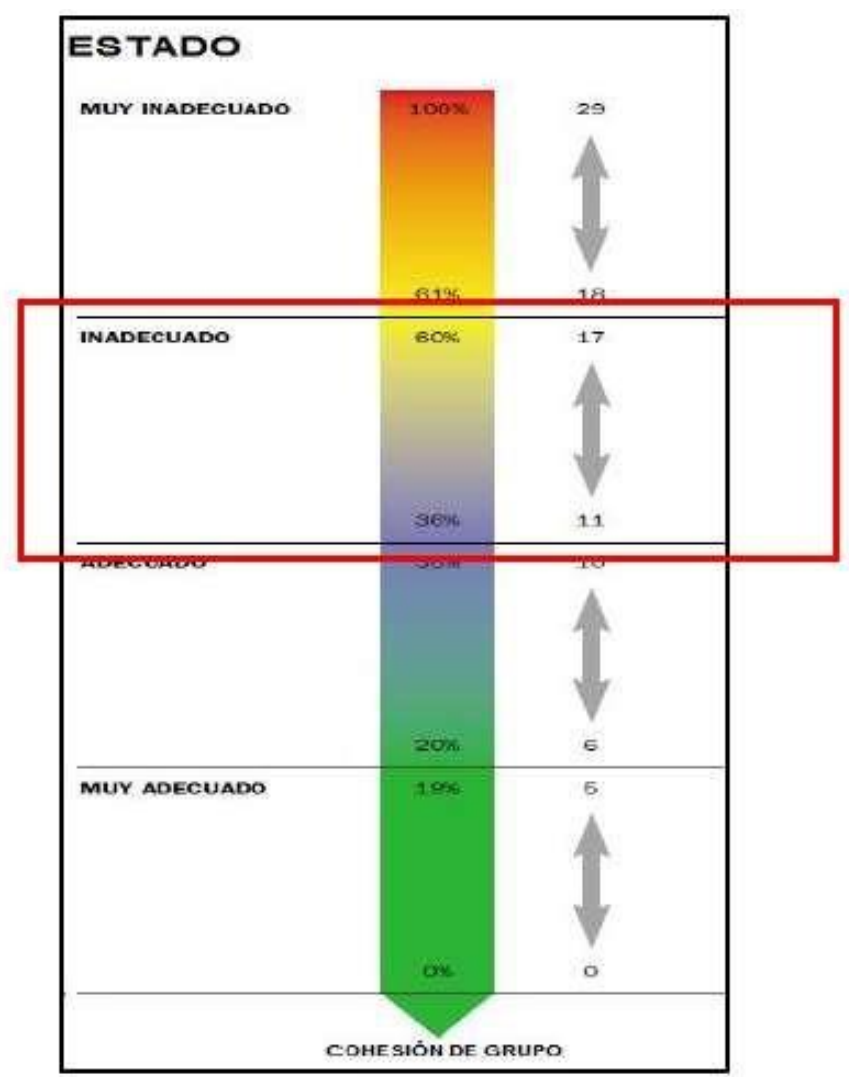

Gráfico 4. Estado Dimensión 4

Fuente: Tabla 6 


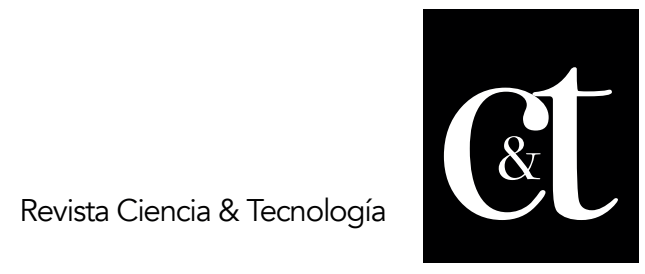

No. 17, 31 de enero de 2018

ISSN impreso: 1390 - 6321

La cohesión determina la relación que surge entre los miembros de la institución, la creación de grupos de trabajo y su participación activa en las actividades planificadas. En esta dimensión involucra aspectos como la solidaridad, atracción, ética y clima laboral. De los 96 colaboradores encuestados, 74 colaboradores equivalente al $77 \%$ se encuentran en el estado inadecuado, 19 colaboradores correspondientes al $20 \%$ están en el estado adecuado; mientras tanto que 3 colaboradores equivalente al $3 \%$ se encuentran en el estado muy adecuado.

Los resultados evidencian una inadecuada cohesión de grupo que limita la coordinación de esfuerzo para obtener objetivos comunes entre los distintos equipos de trabajo y niveles jerárquicos verticales y horizontales; existiendo una deficiente cooperación y apoyo laboral; generando ambientes de trabajo estresantes que afecta la eficiencia grupal e individual.

\section{Verificación de Hipótesis}

Se construyó el modelo lógico partiendo de la hipótesis

$\mathrm{HO}=$ La comunicación interna NO se vincula con la eficiencia laboral del personal de CE del Hospital General Docente Ambato.

$\mathrm{H} 1=$ La comunicación interna SI se vincula con la eficiencia laboral del personal de CE del Hospital General Docente Ambato.

\section{Modelo Matemático}

Hipótesis nula $\mathrm{HO}=$ Respuestas observadas = Respuestas Esperadas

Hipótesis alternativa $\mathrm{H} 1=$ Respuestas observadas $\neq$ Respuestas esperadas.

\section{Nivel de significación}

La probabilidad de rechazar la hipótesis nula cuando es falsa es de $5 \%$, es decir, el nivel de confianza es del $95 \%$.

\section{Estadístico de prueba}

Para la verificación de la hipótesis se toma la fórmula del Chi cuadrado, se utilizaron los resultados del Test Factores Psicosociales de las dimensiones: Participación, Implicación, Responsabilidad; Formación, Información y Comunicación; Gestión del Tiempo; Cohesión de Grupo.

De las cuatro dimensiones se obtuvieron las siguientes tablas: 
Tabla 7. Respuestas observadas

\begin{tabular}{|l|l|l|l|l|l|}
\hline DIMENSIONES & $\begin{array}{l}\text { Muy } \\
\text { inadecuado }\end{array}$ & Inadecuado & Adecuado & $\begin{array}{l}\text { Muy } \\
\text { adecuado }\end{array}$ & TOTAL \\
\hline $\begin{array}{l}\text { Participación, } \\
\text { Implicación } \\
\text { Responsabilidad }\end{array}$ & 0 & 46 & 39 & 11 & 96 \\
\hline $\begin{array}{l}\text { Formación, Información } \\
\text { Comunicación }\end{array}$ & 19 & 48 & 18 & 11 & 96 \\
\hline Gestión del tiempo & 22 & 39 & 26 & 9 & 96 \\
\hline Cohesión de grupo & 0 & 74 & 19 & 3 & 96 \\
\hline TOTAL & $\mathbf{4 1}$ & $\mathbf{2 0 7}$ & 102 & $\mathbf{3 4}$ & $\mathbf{3 8 4}$ \\
\hline
\end{tabular}

Fuente: Test Factores Psicosociales

Tabla 8. Respuestas esperadas

\begin{tabular}{|c|c|c|c|c|c|}
\hline DIMENSIONES & $\begin{array}{l}\text { Muy } \\
\text { inadecuado }\end{array}$ & Inadecuado & Adecuado & $\begin{array}{l}\text { Muy } \\
\text { adecuado }\end{array}$ & TOTAL \\
\hline $\begin{array}{ll}\text { Participación, } & \text { Implicación } \\
\text { Responsabilidad } & \end{array}$ & 10,25 & 51,75 & 25,5 & 8,5 & 96 \\
\hline $\begin{array}{ll}\text { Formación, } & \text { Información } \\
\text { comunicación } & \end{array}$ & 10,25 & 51,75 & 25,5 & 8,5 & 96 \\
\hline Gestión del tiempo & 10,25 & 51,75 & 25,5 & 8,5 & 96 \\
\hline Cohesión de grupo & 10,25 & 51,75 & 25,5 & 8,5 & 96 \\
\hline TOTAL & 41 & 207 & 102 & 34 & 384 \\
\hline
\end{tabular}

Fuente: Test Factores Psicosociales

En base a los valores obtenidos en las tablas 7 y 8 , se aplicaron las siguientes fórmulas para obtener el valor del estadístico Chi-cuadrado y poder verificar la hipótesis formulada: 


$$
X^{2}=\frac{\sum(O-E)^{2}}{E}
$$

$\mathrm{X}^{2}=$ Valor a calcularse de Chi-cuadrado

$\Sigma=$ Sumatoria

$\mathrm{O}=$ Respuestas observadas de la investigación

$\mathrm{E}=$ Respuestas esperadas o calculadas

Regla de decisión

Si $x^{2} c>x^{2} t$, rechazo $H 0$ y acepto $H 1$, en este caso se trabajó con 4 filas y 4 columnas, dando como resultado a 9 grados de libertad $(g l=9)$ y se corroboró el valor $x^{2} t$, es igual a 16,91 con un nivel de significación de $5 \%$.

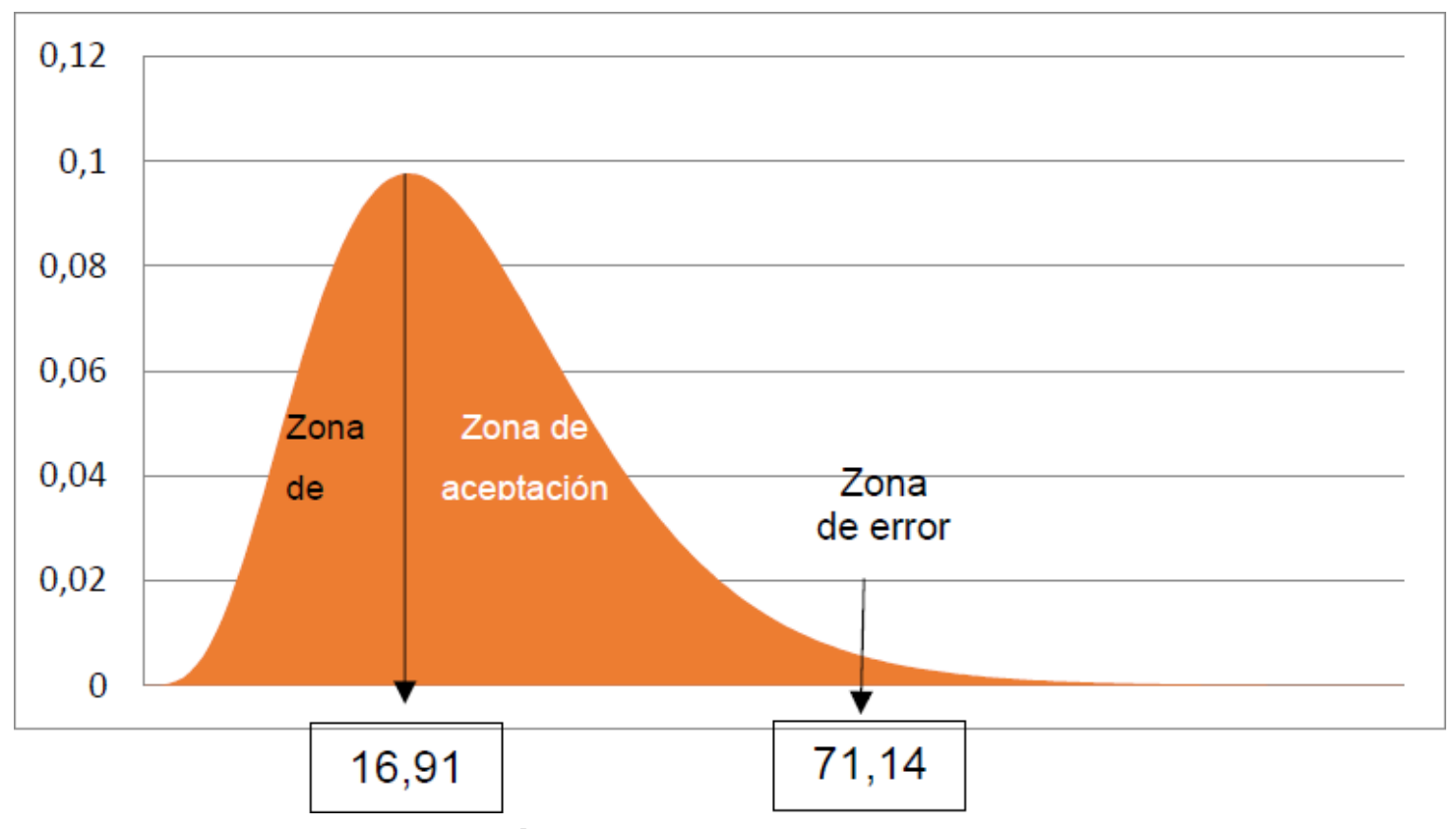

Gráfico 5. Campana de Gauss

El valor de $x^{2} c=71,14>x^{2} t=16,919$ y de conformidad a lo establecido en la regla de decisión, se rechaza la hipótesis nula y se acepta la hipótesis alternativa, es decir, se confirma que: La comunicación interna sí se vincula con la eficiencia laboral del personal de Consulta Externa del Hospital General Docente Ambato.

\section{Discusión}

La correlación establece la relación o dependencia existente entre dos variables con el fin de determinar si los cambios en una de las variables influyen en los cambios de 


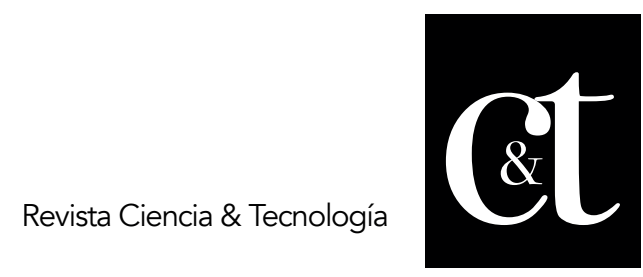

No. 17, 31 de enero de 2018

ISSN impreso: 1390 - 6321

la otra; en tal caso, se dice que existe correlación entre ellas.

Inicialmente se representó la variable independiente con los resultados obtenidos de la Dimensión 2 del Test de Factores Psicosociales de Identificación de Situaciones de Riesgo: Formación, Información y Comunicación. Asimismo, la variable dependiente con la dimensión 3: Gestión del Tiempo.

Estos resultados fueron representados en un gráfico de dispersión con el objeto de determinar el tipo de relación existente entre las variables. El siguiente gráfico muestra la dirección de la relación entre las variables positiva, demostrando una correlación lineal directa.

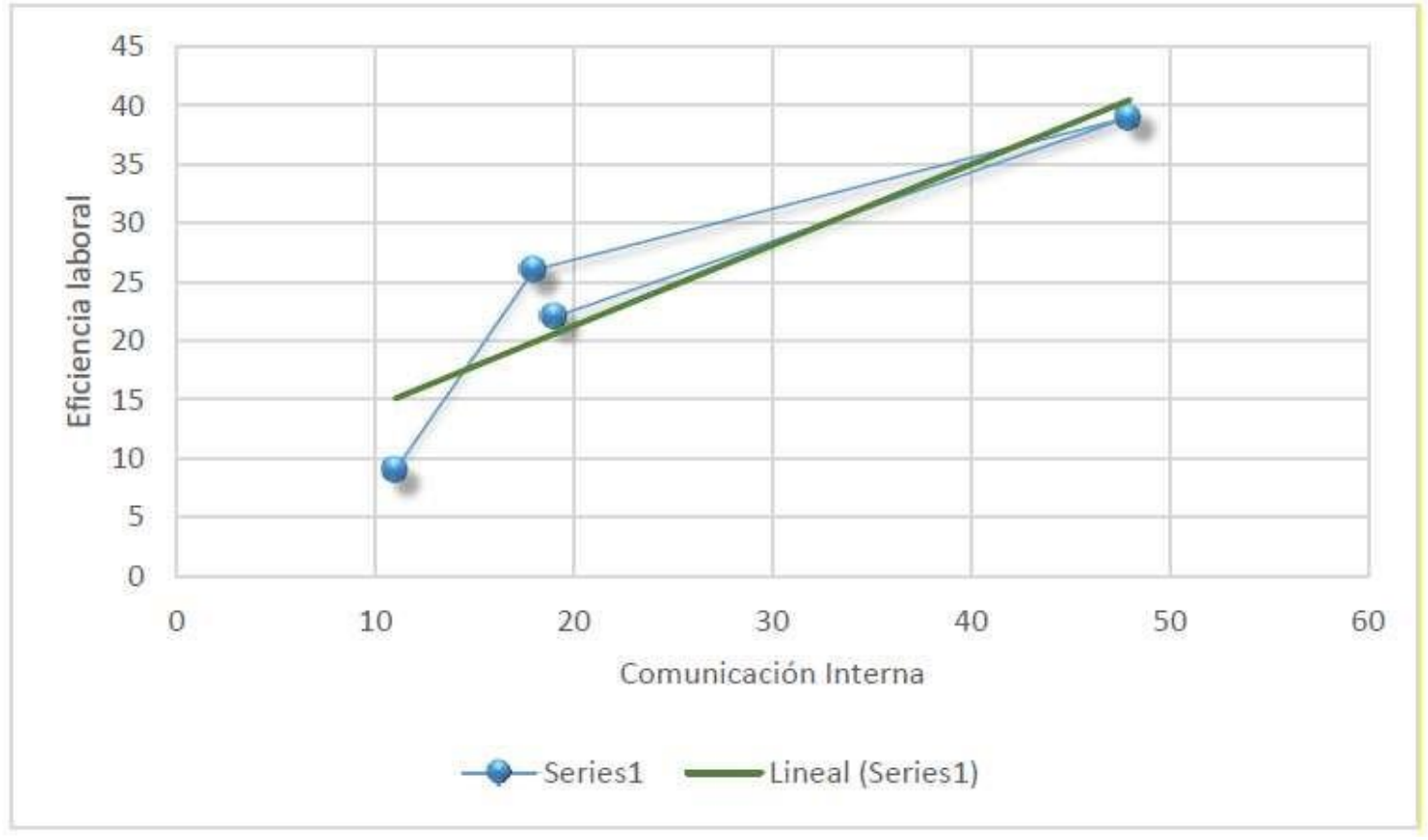

Gráfico 6. Gráfico de relación de las variables

Los valores de la Tabla 8 calculan el coeficiente de correlación lineal tomando los valores de la Dimensión 2: Formación, Información, Comunicación y, Dimensión 3: Gestión del Tiempo correspondiente al Test de Factores Psicosociales de Navarra aplicado a los colaboradores del área de CE del Hospital General Docente Ambato, con el estado: muy inadecuado, inadecuado, adecuado y muy adecuado.

Al ser el coeficiente de correlación positivo, la correlación es directa y por encontrarse muy próximo a 1 , la correlación es muy fuerte; es decir, que cuando aumenten los valores de la variable comunicación interna también aumentarán los valores de la variable eficiencia laboral.

\section{Conclusiones}

La dimensión participación, implicación, responsabilidad detalla el grado de libertad 
e independencia que tiene el trabajador para controlar, organizar su trabajo y determinar los métodos a utilizar.

De esta manera, 46 colaboradores que corresponden al $48 \%$ se encuentran en el estado inadecuado del Test de Factores Psicosociales; mientras que apenas 11 colaboradores equivalente al $11 \%$ están en el estado muy adecuado.

Consecuentemente, los colaboradores encuestados evidencian un estado inadecuado en la dimensión autonomía, trabajo en equipo, iniciativa, control sobre la tarea, rotación, supervisión y enriquecimiento de tareas lo cual repercute en la eficiencia de los colaboradores.

Es decir, no se encuentra grado de independencia y libertad que tiene el colaborador para controlar y organizar su propio trabajo, además de determinar los métodos a utilizar. No existe autonomía en su trabajo, y eso mata la iniciativa y creatividad en las funciones diarias.

La mayoría de colaboradores evidencia una inadecuada formación, información y comunicación interna como consecuencia del deficiente uso de medios de comunicación interna, desconocimiento de perfiles de cargos según niveles jerárquicos, fuertes flujos de comunicación informal y escasa difusión de la planificación institucional.

El hospital muestra un desinterés total por los trabajadores dificultando el flujo de información para la eficiente actividad, indirectamente y/o contribuciones de cada colaborador está bien definida, esto se debe a las deficiencias existentes en el manual de funciones y procesos internos.

De la misma manera, 22 personas equivalente al $23 \%$ se encuentran en el nivel muy inadecuado, mientras que 9 colaboradores representan el $9 \%$ están en el estado muy adecuado de la dimensión 3 gestión del tiempo.

Los colaboradores que se encuentran en el estado muy adecuado son los líderes departamentales. Que su ritmo de trabajo y carga son planificados por ellos mismo, tal vez sería recomendable hacer un estudio de tiempos y movimientos para la medición de fusiones y tareas según el diseño de puesto estructurado en el hospital. A breves rasgos, la distribución de las pausas y la elección de las vacaciones de acuerdo a sus necesidades personales, no determina el empleado, sino, que es impuesto.

La cohesión determina la relación que surge entre los miembros de la institución, la creación de grupos de trabajo y su participación activa en las actividades planificadas. En esta dimensión involucra aspectos como la solidaridad, atracción, ética y clima laboral.

Los resultados evidencian una inadecuada cohesión de grupo que limita la coordinación de esfuerzo para obtener objetivos comunes entre los distintos equipos de trabajo y niveles jerárquicos verticales y horizontales; existiendo una deficiente cooperación y apoyo laboral; generando ambientes de trabajo estresantes que afecta la eficiencia grupal e individual. 


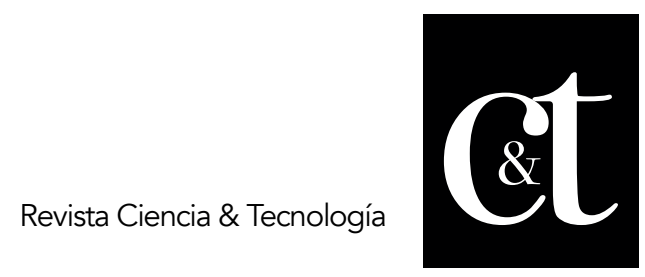

No. 17, 31 de enero de 2018

ISSN impreso: 1390 - 6321

\section{Referencias bibliográficas}

Albornoz, M. (2014). Las relaciones humanas en el ámbito laboral. Obtenido de http://mayeuticaeducativa.idoneos.com/relaciones del trabajo/

Altamirano, M. (2014). La Comunicación en el Hospital Metropolitano: Mecanismos para el análisis de la comunicación. Tesis de Licenciatura, Universidad San Francisco de Quito, Colegio de Comunicación y Artes Contemporáneas. Obtenido de Repositorio de la Universidad San Francisco de Quito: http://repositorio.usfq.edu.ec/handle/23000/3223

Andrade, H. (2005). Comunicación Organizacional Interna: proceso, disciplina, técnica. España: Netbiblo.

Bastida, R. (2013). Factores de riesgo psicosocial a través del método FPSICO en una de automoción de Navarra. Navarra: UPNA.

Banco Central del Ecuador BCE (2010). País en lugar 137 de eficiencia laboral. Obtenido de http://biblioteca.bce.ec/cgi-bin/koha/opacdetail.pl?biblionumber $=91193$

Chimborazo, L. (2013). La comunicación interna y externa y su incidencia en el desarrollo de la imagen corporativa de Industrias Catedral S. A. en la ciudad de Ambato. Tesis de Maestría. Universidad Técnica de Ambato. Obtenido de http://redi.uta.edu.ec/bitstream/123456789/6378/1/60MBA.pdf

Chinchillas, D. (2011). El flujo de la comunicación en la organización. Obtenido de http://biblioteca.itson. $\mathrm{mx} / \mathrm{oa} / \mathrm{ciencias}$ administrativa/oa19/comunicacion direc cion naturaleza proyecto/z2.htm

Daft, R. y Marcic, D. (2010). Introducción a la Administración. México: Cengage Learning.

Del Prado, L. (2016). Marketing Interno. Obtenido de http://200.16.86.50/digital/33/revistas/blse/delprado7-7.pdf

Druker, P. (2000). La gerencia en tiempos difíciles. México: Continental SA de CV.

Formanchuk, A. (2010). Comunicación interna, comunicación interpersonal. Recuperado el 20 de Agosto de 2015, de http://formanchuk.com.ar/todosignifica/mala- comunicación/

Gómez, M. (2006). Introducción a la Metodología de la Investigación Científica. Córdova: Brujas.

Guardia, J. (2008). Evaluación de los principales métodos de evaluación de riesgos psicosociales. Barcelona: Universidad de Barcelona.

Herrero, L. (2015). Eficiencia técnica de los hospitales públicos y empresas públicas hospitalarias de Andalucía. Gaceta Sanitaria, Vol. 29. Núm. 4: 274-281.

Kompier, M y Levi, L. (1994). Estrés en el trabajo: causas, efectos y prevención. Fundación Europea para la Mejora de las Condiciones de Vida y de Trabajo. Dublín: Fundación Europea.

Lahera, M., \& y Góngora, J. J. (2002). Factores Psicosociales. Gobierno de Navarra: Instituto Navarro de Salud Laboral. 


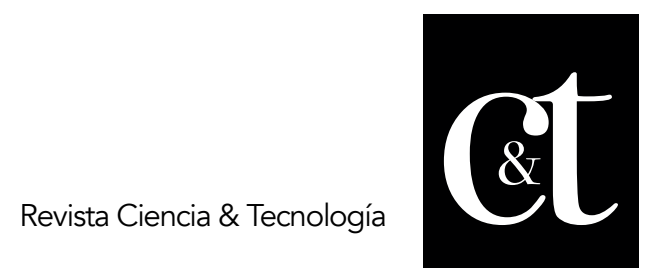

No. 17, 31 de enero de 2018

ISSN impreso: 1390 - 6321

Medina, P. (2012). El valor estratégico de la comunicación interna hospitalaria. Revista de Comunicación y Salud, 19-28.

Mira, J. (2013). La comunicación interna en el hospital. Unidades Docentes de la Escuela Nacional de Sanidad. UNEDENSISCIII, 31.

Nogareda, C. (2009). El método del INSL para identificación y evaluación de factores psicosociales. Navarra: Instituto Nacional de Seguridad e Higiene en el Trabajo.

Ocampo, M. ed. (2011). Comunicación empresarial. Plan Estratégico como herramienta gerencial y nuevos retos del comunicador en las organizaciones. Bogotá: ECOE.

Pereira, A. (2003). Llamar la atención sin romper el clima. Buenos Aires: AP Consultores.

Pilonieta, G. (2007). Desarrollo Pedagógico, Investigación, aprendizaje y gestión organizacional. Obtenido de http://www.cisne.org/Aprendizajeexperiencial/

Quino, I. (2014). Comunicación Interna. Obtenido de http://www.hospitalharrywilliams.org/manuales/comunicacion-internaestudio/

Quintero, Y. (2009). Talento Humano. Obtenido de http://equipo4-rrhh-unesr.overblog.es/article-33672212.html

Ramos, M. (2011). La motivación laboral y su relación con el clima laboral en los trabajadores del Banco Ecuatoriano de la Vivienda Matriz Quito. Tesis de grado, Universidad Central del Ecuador. Obtenido de http://www.dspace.uce.edu.ec/bitstream/25000/1897/1/T-UCE-0007-24.pdf

Rebeil, M. A. (2010). El poder de la comunicación en las organizaciones. México: Plaza y Valdés Editores.

Reina, M. (2015). La importancia del capital humano. Obtenido de http://www.portafolio.co/columnistas/la-importancia-del-capital-humanoopinion

Reyes, A. (2003). Administración Moderna. México: Grupo Noriega.

Secretaría Nacional de la Administración Pública (2015). Sistema de Gestión Documental Quipux. Obtenido de http://www.administracionpublica.gob.ec/sgdq-sistema-de-gestiondocumental-quipux/

Suing, A. (2012). Gestión en Comunicación: investigación aplicada, extensión y servicios. Obtenido de Comunicación interna para las organizaciones ecuatorianas: https://abelsuing.wordpress.com/2012/12/21/comunicacioninterna-para-las- organizaciones-ecuatorianas/\#comments 